MOURA, M.S. et al. Análise do comportamento de Callithrix jacchus Linnaeus, 1758 do zoológico de Uberlândia-MG. PUBVET, Londrina, V. 5, N. 4, Ed. 151, Art. 1016, 2011.

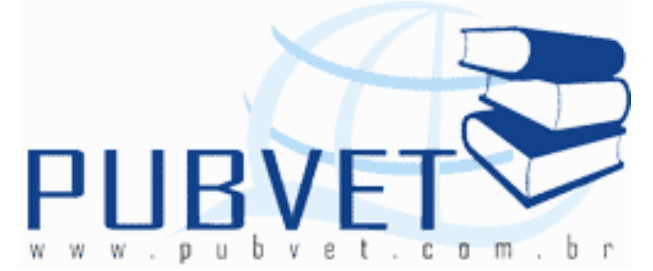

PUBVET, Publicações em Medicina Veterinária e Zootecnia.

\title{
Análise do comportamento de Callithrix jacchus Linnaeus, 1758 do zoológico de Uberlândia-MG
}

Mariela Silva Moura ${ }^{1 *}$, Fernanda Silva Ferreira ${ }^{1}$, Fabiane Vieira de Oliveira ${ }^{1}$,
Thalita Rocha Brito ${ }^{1}$, André Luiz Quagliatto Santos ${ }^{2}$, Líria Queiroz Luz Hirano ${ }^{3}$

${ }^{1}$ Graduanda em Medicina Veterinária, Universidade Federal de Uberlândia. Uberlândia, MG.

${ }^{2}$ Docente do Programa de Pós-graduação em Ciências Veterinárias, Universidade Federal de Uberlândia. Uberlândia, MG.

${ }^{3}$ Mestranda em Ciências Veterinárias, Universidade Federal de Uberlândia. Uberlândia, MG.

*Autor para correspondência: Rua Ceará, s/n, Bloco 2D, CEP 38400-902, Uberlândia, MG, Brasil. E-mail: marielasmoura@yahoo.com.br

\section{Resumo}

Os zoológicos foram criados com o propósito de promover o bem estar e a conservação das espécies, entretanto, animais cativos podem apresentar comportamentos discrepantes de seu padrão natural. Esse trabalho foi desenvolvido com o intuito de descrever o comportamento de saguis de tufo branco Callithrix jacchus, do zoológico de Uberlândia, MG. Na tentativa de simular o habitat natural, o recinto dos animais é equipado com objetos para o enriquecimento ambiental. O método amostral utilizado foi o ad libitum, durante o período de dois dias, com dez horas diárias e anotações 
MOURA, M.S. et al. Análise do comportamento de Callithrix jacchus Linnaeus, 1758 do zoológico de Uberlândia-MG. PUBVET, Londrina, V. 5, N. 4, Ed. 151, Art. 1016, 2011.

comportamentais em intervalos de cinco minutos. As atividades mais realizadas durante as observações de Callithrix jacchus foram a alimentação, descanso e brincar. Muitos aspectos do padrão de atividade do grupo foram semelhantes àqueles que habitam outras formações vegetais, inclusive no habitat natural do animal. Isso sugere que esses primatas possuem certa capacidade de se adaptarem aos mais variados tipos de habitat, inclusive o cativeiro.

Palavras-chave: primatas, comportamento animal, sagui de tufo branco

\section{Analysis of the behavior of Callithrix jacchus in zoo of Uberlândia-MG}

\section{Abstract}

Zoos were created to promote the welfare and conservation of species, however, animals living in captivity may exhibit behaviors outside the standard of free living. It has been proposed as objective make the behavioral analysis of common marmoset Callithrix jacchus, in a zoo located in Uberlândia, MG, Brazil. In the animals captivity were found objects used as environmental enrichment, which tried to simulate a natural habitat. Using the ad libitum method, the observation period was divided into two days with ten hours each, with annotations behavior at intervals of five minutes. The activities more performed during the observations of Callithrix jacchus were eat, rest and play. Many aspects of the activity pattern of the group were similar to those inhabiting other places, including the natural habitat of the animals. This suggests that these primates have some capacity to adapt to a variety of habitat types, including captivity.

Keywords: primates, animal behavior, common marmoset

\section{Introdução}

Dentre os gêneros de calitriquíneos, o Saguinus e o Callithrix são os mais comumente criados em cativeiro para uso em pesquisas biomédicas (HERSHKOVITZ, 1977). A espécie Callithrix jacchus, conhecida popularmente 
MOURA, M.S. et al. Análise do comportamento de Callithrix jacchus Linnaeus, 1758 do zoológico de Uberlândia-MG. PUBVET, Londrina, V. 5, N. 4, Ed. 151, Art. 1016, 2011.

como sagui de tufo branco, habita principalmente as regiões de caatinga e cerrado brasileiro. São animais de pequeno porte com o peso entre 350 e 450 gramas, pelagem estriada nas orelhas e mancha branca na testa. Esses primatas de hábitos diurnos atingem a maturidade sexual com aproximadamente 13 meses e alimentam-se de frutas, verduras, sementes, flores, artrópodes e filhotes de aves (AURICCHIO, 1995).

No ambiente natural, os animais são submetidos a desafios e perigos que exigem um amplo repertório comportamental e cada espécie possui habilidades específicas que evoluíram em função de seu nicho ecológico (BAYNE, 1991). Em contrapartida, o cativeiro geralmente possui um espaço físico reduzido e empobrecido, o que pode resultar em comportamentos indesejáveis com o aumento do estresse e da agressividade (ANDRADE, 2000).

De acordo com Anderson e Visalberghi (1991), o bem-estar psicológico dos primatas pode ser avaliado com base na ausência de estresse e de comportamento estereotipado, na capacidade de lidar com as mudanças ambientais e sociais e na presença de um amplo repertório comportamental.

Várias estratégias têm sido utilizadas para o enriquecimento de ambientes cativos de primatas. Bloomsmith et al. (1990) disponibilizaram bolas plásticas rígidas para chimpanzés e observaram uma maior disposição dos animais às atividades físicas, o que combateu o tédio e a obesidade. Em adjunto, o uso de uma tábua de forrageio por Bayne et al. (1991) reduziu significantemente os níveis de comportamentos anormais em macacos-resos mantidos em isolamento.

Esse trabalho foi desenvolvido com o intuito de descrever o comportamento de Callithrix jacchus, do zoológico de Uberlândia, MG.

\section{Material e métodos}

A observação dos dados foi realizada em um zoológico da cidade de Uberlândia, MG. Nesse local, o recinto dos saguis de tufo branco foi construído com tijolos, possui piso de cimento e uma grade metálica que possibilita a visualização de seu interior. 
MOURA, M.S. et al. Análise do comportamento de Callithrix jacchus Linnaeus, 1758 do zoológico de Uberlândia-MG. PUBVET, Londrina, V. 5, N. 4, Ed. 151, Art. 1016, 2011.

A jaula de Callithrix jacchus encontra-se próxima a um escritório administrativo da instituição e é composta por arbustos, galhos, um chafariz que se encontrava inoperante, uma toca feita com pedras rústicas e alguns objetos para o enriquecimento ambiental, dentre eles: uma cabaça e cachos de coquinhos que também servem como alimento. A comida dos animais é servida em uma vasilha de plástico que fica no chão do recinto, próximo à casa de pedras.

Foram analisados os comportamentos dos animais sem distinção de idade e sexo e padronizaram-se os dados em 13 tipos principais de comportamentos para os primatas da jaula. Para a confecção dos padrões comportamentais foi utilizada a técnica de observação ad libitum, método no qual se observa continuamente o exemplar e se registra toda nova atividade (ALTMAN, 1974).

Anotações das atitudes dos animais foram feitas em intervalos de aproximadamente cinco minutos, durante dois dias, em dez horas diárias. Visando captar um maior número de comportamentos, as observações foram feitas em diferentes horas do dia.

Aplicou-se a técnica de observação denominada animal focal, na qual se analisa o exemplar em um curto período de tempo, suficiente para se avaliar alguns comportamentos, diferentes ou repetidos. Os dados foram submetidos para análise no programa Microsoft Excel $2003^{\circledR}$.

\section{Resultados e Discussão}

Dentre as atividades promovidas pelos saguis, observaram-s: brincar, descansar, andar pela jaula, comunicar com a jaula vizinha, vocalizar, andar na grade, atividade sexual, espreguiçar na parede, agitação devido à presença de sagüis soltos, higienização, banho de sol e comer. A frequência de observação desses comportamentos encontra-se no Quadro 1.

De acordo com Bicca-Marques et al. (2006), os saguis soltos em seu habitat natural são agitados e brincalhões, o que se repete dentro de um cativeiro. No zoológico de Uberlândia, esses animais são estimulados a se 
MOURA, M.S. et al. Análise do comportamento de Callithrix jacchus Linnaeus, 1758 do zoológico de Uberlândia-MG. PUBVET, Londrina, V. 5, N. 4, Ed. 151, Art. 1016, 2011.

exercitarem através da disposição de galhos e outros aparatos por todo o recinto.

Dentro das 20 horas de observação da espécie Callithrix jacchus, não se observaram comportamentos indicativos de pacing, ou seja, locomoção dos exemplares de um lado para outro do recinto. Adicionalmente, não foram observadas atividades indicativas de estresse, contudo, algumas melhoras podem ser feitas visando uma melhor qualidade de vida para esses animais.

Quadro 1 - Padrões e frequências (\%) de atividades observados em saguis de tufo branco do zoológico de Uberlândia, MG

\begin{tabular}{|c|c|c|c|c|c|c|c|c|c|c|c|c|c|}
\hline \multicolumn{1}{|c|}{ REPETIÇÕES DE COMPORTAMENTO } \\
\hline & A & B & C & D & E & F & G & H & I & J & K & L & M \\
\hline$\%$ & 18,73 & 14,23 & 15,73 & 6,37 & 9,36 & 8,61 & 9,36 & 4,87 & 2,25 & 2,62 & 3 & 4,12 & 0,75 \\
\hline
\end{tabular}

Legenda: A - Comer; B - Brincar; C - Descansar; D - Andar; E - Comunicação com a jaula ao lado; F - Vocalização; G - Na grade; H - Atividade sexual; I Espreguiçar na parede; J - Agitação devido à visita de saguis soltos; K Higienização; L - Banho de sol; M - Entrar na casa

No Gráfico 2, observa-se a frequência de alimentação dos animais. Nota-se que os saguis comem com maior constância no período da manhã e que em praticamente todas as horas de avaliação houve presença dessa atividade, o que reforça a necessidade do enriquecimento dos recintos de modo a estimular os animais a realizarem atividades físicas para evitar problemas de saúde, como a obesidade.

Em concordância com o presente estudo, Alonso e Langguth (1989) observaram que a atividade de alimentação foi predominante no padrão comportamental de Callithrix jacchus, com $27 \%$ de frequência. Entretanto, Cavalcanti (2002) relata que na mata atlântica os grupos dessa espécie investem mais tempo em forrageio (50\%), com ingestão de alimentos (15\%) conseguidos nessa procura. Além disso, o descanso foi uma das três atividades 
MOURA, M.S. et al. Análise do comportamento de Callithrix jacchus Linnaeus, 1758 do zoológico de Uberlândia-MG. PUBVET, Londrina, V. 5, N. 4, Ed. 151, Art. 1016, 2011.

mais realizadas durante a observação, o que também foi relatado em relação a dois dos três grupos de C. jacchus estudados por Casimiro (2003).

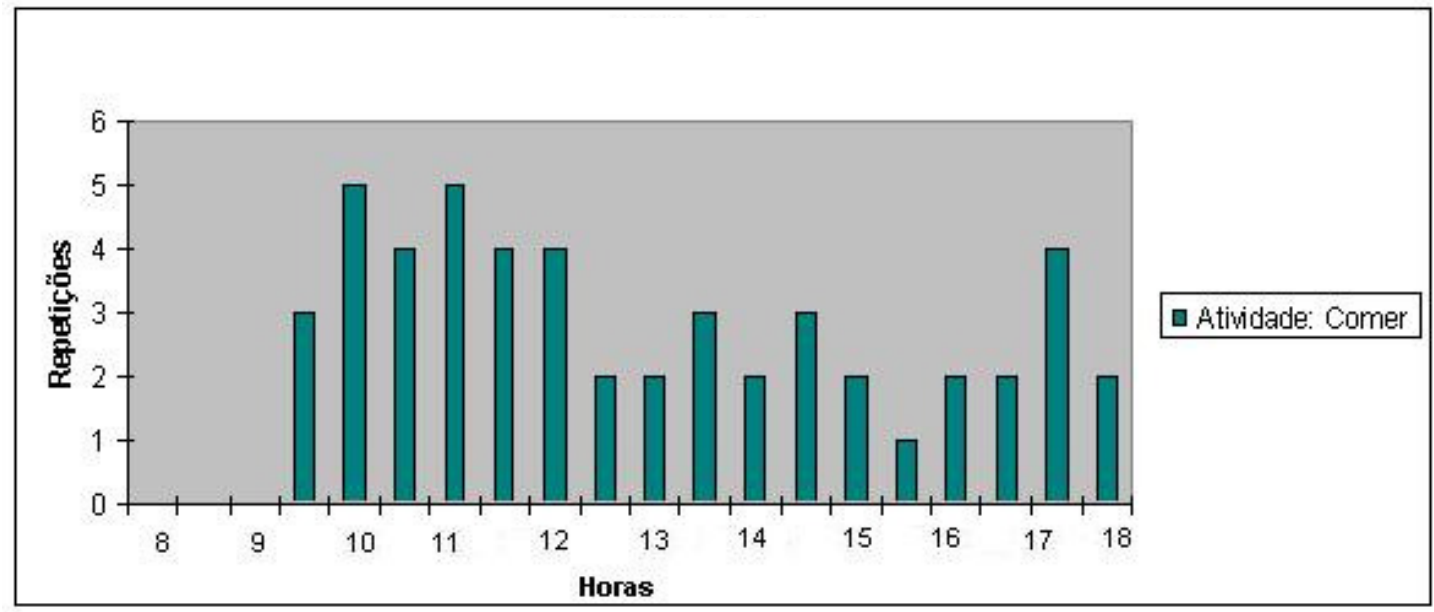

Gráfico1 - Frequência alimentar de sagüis de tufo branco do zoológico de Uberlândia, MG

\section{Conclusão}

As atividades mais realizadas pelos Callithrix jacchus em cativeiro foram alimentação, descanso e brincar. Adicionalmente, muitos aspectos do padrão de atividade do grupo foram semelhantes àqueles que habitam outras formações vegetais, inclusive no habitat natural do animal, o que sugere que esses primatas possuem certa capacidade de se adaptarem aos mais variados tipos de habitat, inclusive o cativeiro.

\section{Referências}

ALONSO, C.; LANGGUTH, A. Ecologia e comportamento de Callithrix jacchus. (Primates: Callitrichidae) numa ilha de Floresta Atlântica. Revista Nordestina de Biologia, João Pessoa, v. 6, n. 2, p. 105-137, 1989.

ALTMANN, J. Observational study of behavior: Sampling methods. Behaviour, Leiden, v. 49, n. 4, p. 227-267, 1974. 
MOURA, M.S. et al. Análise do comportamento de Callithrix jacchus Linnaeus, 1758 do zoológico de Uberlândia-MG. PUBVET, Londrina, V. 5, N. 4, Ed. 151, Art. 1016, 2011.

ANDERSON, J.R.; VISALBERGHI, E. Primate psychological well-being: a comparative approach to environmental enrichment for captive primates. Applied Animal Behaviour Science, Amsterdam, v. 30, n. 1-2, p. 195, 1991.

ANDRADE, R. F. Sugestão para o aprimoramento das condições ambientais e promoção do bem estar em macacos pregos em cativeiro (Cebus apella, Plathyrrhini, Cebidae). 2000. Trabalho de Conclusão de Curso - Centro de Ciências Biológicas, Universidade Federal do Pará, Belém, 2000.

AURICCHIO, P. Primatas do Brasil. São Paulo: Terra Brasilis, 1995. 168p.

BAYNE, K. Providing environmental enrichmental to captive primates. Small Animal, Kansas, v. 13, n. 11 , p. 1689-1695, 1991.

BAYNE, K.; MAINZER, H.; DEXTER, S.; CAMPBELL, G.; YAMADA, F.; SUOMI, S. The reduction of abnormal behaviors in individually housed rhesus monkeys (Macaca mulatta) with a foraging/grooming board. American Journal of Primatology, New York, v. 23, p. 23-35, 1991.

BLOOMSMITH, M. A.; FINLAY, T. W.; MERHALSKI, J. J.; MAPLE T. L. Rigid plastic balls as enrichment devices for captive chimpanzees. Laboratory Animal Science, Memphis, v. 40, n. 3, p. 319-322, 1990.

BICCA-MARQUES, J. C; SILVA, V. M.; GOMES, D. F. Ordem Primates. In: REIS, N. R.; PERACCHI, A. L.; PEDRO, W. A.; LIMA, I. P. Mamíferos do Brasil. Londrina, Paraná. CD-ROM. 2006.

CASIMIRO, R. F. Utilização da área domiciliar por grupos de saguis Callithrix jacchus Linneus, 1758 (Primates: Callithrichidae). 2003. 52 f. Dissertação (Mestrado)Universidade Federal do Rio Grande do Norte, Natal, 2003.

CAVALCANTI, K. C. S. Comportamento alimentar de Callithrix jacchus (Callitrichidae: Primates) em mata secundária e ambiente alterado. 2002. 48 f. Dissertação (Mestrado)Universidade Federal do Rio Grande do Norte, Natal, 2002.

HERSHKOVITZ, P. Living New World Monkeys. Chicago: Chicago University Press, p. 701703, 1977. 